\title{
Fluoxetine (Prozac) Binding to Serotonin Transporter Is Modulated by Chloride and Conformational Changes
}

\author{
Sotiria Tavoulari, ${ }^{1}$ Lucy R. Forrest, ${ }^{2}$ and Gary Rudnick ${ }^{1}$ \\ ${ }^{1}$ Department of Pharmacology, Yale University School of Medicine, New Haven, Connecticut 06520-8066, and ${ }^{2}$ Computational Structural Biology Group, \\ Max Planck Institute of Biophysics, 60438 Frankfurt am Main, Germany
}

Serotonin transporter (SERT) is the main target for widely used antidepressant agents. Several of these drugs, including imipramine, citalopram, sertraline, and fluoxetine (Prozac), bound more avidly to SERT in the presence of $\mathrm{Cl}^{-}$. In contrast, $\mathrm{Cl}^{-}$did not enhance cocaine or paroxetine binding. $\mathrm{A} \mathrm{Cl}^{-}$binding site recently identified in SERT, and shown to be important for $\mathrm{Cl}^{-}$dependent transport, was also critical for the $\mathrm{Cl}^{-}$dependence of antidepressant affinity. Mutation of the residues contributing to this site eliminated the $\mathrm{Cl}^{-}$-mediated affinity increase for imipramine and fluoxetine. Analysis of ligand docking to a single state of SERT indicated only small differences in the energy of interaction between bound ligands and $\mathrm{Cl}^{-}$. These differences in interaction energy cannot account for the affinity differences observed for $\mathrm{Cl}^{-}$dependence. However, fluoxetine binding led to a conformational change, detected by cysteine accessibility experiments, that was qualitatively different from that induced by cocaine or other ligands. Given the known $\mathrm{Cl}^{-}$requirement for serotonin-induced conformational changes, we propose that $\mathrm{Cl}^{-}$binding facilitates conformational changes required for optimal binding of fluoxetine and other antidepressant drugs.

\section{Introduction}

Serotonin transporter (SERT) is responsible for reuptake of the neurotransmitter serotonin [5-hydroxytryptamine (5-HT)] into neurons after its release (Rudnick, 2006). SERT belongs to the neurotransmitter sodium symporter (NSS) (SLC6) family of transporters that also includes transporters for dopamine (DAT), norepinephrine (NET), glycine, and GABA. It is a target for drugs of abuse, including cocaine and 3,4-methylenedioxymethamphetamine (ecstasy) (Rudnick and Wall, 1992). Furthermore, SERT is associated with several psychiatric disorders, such as major depression, anxiety, and obsessive compulsive disorder (Murphy et al., 2004), and represents the primary target for widely used antidepressants, including fluoxetine (Prozac), sertraline (Zoloft), citalopram (Celexa), and paroxetine (Paxil). Although computational and mutagenesis approaches have identified binding sites for substrates and cocaine (Beuming et al., 2008; Celik et al., 2008; Kaufmann et al., 2009), molecular determinants of antidepressant interaction with target proteins remain to be identified.

Early studies on imipramine binding to SERT suggested that $\mathrm{Na}^{+}$and $\mathrm{Cl}^{-}$, both required for 5-HT transport, might also determine antidepressant affinity (Talvenheimo et al., 1979; Humphreys et al., 1994). However, binding of cocaine and its analogs (-)-2- $\beta$-carbomethoxy-3 $\beta$-(4-fluorophenyl) tropane (CFT) and

Received Jan. 27, 2009; revised May 20, 2009; accepted June 26, 2009.

We thank Drs. Ove Wiborg and Birgit Schiøtt for the atomic coordinates of their model of 5-HT bound to SERT, Jose Faraldo-Gómez for useful discussions, and Bret Bessac for help with statistical analyses.

Correspondence on general aspects of this paper should be addressed to Dr. Gary Rudnick, Department of Pharmacology, Yale University, P.0. Box 208066, New Haven, C 06520-8066. E-mail: gary.rudnick@yale.edu.

Correspondence relating to computational modeling and docking should be addressed to Dr. Lucy R. Forrest, Max Planck Institute of Biophysics, Max-von-Laue-Strasse 3, 60438 Frankfurt am Main, Germany. E-mail: lucy.forrest@biophys.mpg.de.

DOI:10.1523/JNEUROSCI.0440-09.2009

Copyright $\odot 2009$ Society for Neuroscience $\quad$ 0270-6474/09/299635-09\$15.00/0

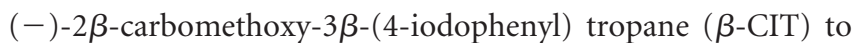
SERT was stimulated only by $\mathrm{Na}^{+}$and not by $\mathrm{Cl}^{-}$(Rudnick and Wall, 1991; Wall et al., 1993). Paroxetine affinity increased with $\mathrm{Na}^{+}$but not $\mathrm{Cl}^{-}$(Cool et al., 1990), and $\mathrm{Na}^{+}$increased SERT affinity for fluoxetine and citalopram (Humphreys et al., 1994; Wong et al., 1995), but the effect of $\mathrm{Cl}^{-}$was not reported. Thus, of all these compounds, only imipramine was shown to bind more avidly to SERT in the presence of $\mathrm{Cl}^{-}$(Talvenheimo et al., 1979; Humphreys et al., 1994). Despite these intriguing differences between SERT inhibitors, the mechanism by which ions stimulate their binding has not yet been elucidated.

As stated above, both $\mathrm{Na}^{+}$and $\mathrm{Cl}^{-}$are absolutely required for 5-HT transport (Lingjaerde, 1969; Sneddon, 1969; Rudnick, 1977). The initial step in 5-HT transport is binding of $5-\mathrm{HT}^{+}$, $\mathrm{Na}^{+}$, and $\mathrm{Cl}^{-}$. Evidence suggests that all three of these solutes must bind before SERT can undergo the conformational changes leading to translocation (Nelson and Rudnick, 1979; Zhang and Rudnick, 2006). The x-ray crystal structure of the amino acid transporter LeuT, a bacterial homolog of SERT, includes two bound sodium ions ( $\mathrm{Na} 1$ and $\mathrm{Na} 2$ ) close to the bound leucine (Yamashita et al., 2005). Evidence for a $\mathrm{Cl}^{-}$site in neurotransmitter transporters has been directly provided by studies with SERT and the GABA transporter GAT-1 (Forrest et al., 2007; Zomot et al., 2007). The proposed $\mathrm{Cl}^{-}$site in SERT is close to the probable Nal site and is formed by residues in transmembrane (TM) helices 2, 6, and 7. Consistent with its role in coupling, $\mathrm{Cl}^{-}$ shares two coordinating residues, Ser336 and Asn368, with the Na1 site (Forrest et al., 2007; Zomot et al., 2007).

Our present work reveals that affinities of several antidepressants (fluoxetine, citalopram, and sertraline) are enhanced by $\mathrm{Cl}^{-}$. We also show that maximal binding of fluoxetine involves formation of a conformational state distinct from that favored by 
cocaine. We propose that $\mathrm{Cl}^{-}$may stimulate fluoxetine binding by facilitating formation of this novel state.

\section{Materials and Methods}

Materials. $\left[{ }^{125} \mathrm{I}\right] \beta$-CIT was purchased from PerkinElmer Life and Analytical Sciences, and 2-aminoethyl methanethiosulfonate hydrobromide (MTSEA) was from Anatrace. Fluoxetine, imipramine, ibogaine, paroxetine, 5 -HT, cocaine, and unlabeled $\beta$-CIT were from Sigma, sertraline from Pfizer, and citalopram from Lundbeck.

Expression of rat SERT mutants and preparation of crude membranes. Site-directed mutagenesis of rat SERT was performed using the Stratagene QuickChange kit. The mutated region was subcloned into rSERT cDNA with a c-Myc epitope tag at the $\mathrm{NH}_{2}$ terminus and a FLAG epitope tag at the $\mathrm{COOH}$ terminus. All mutations were confirmed by DNA sequencing.

The VTF7-3 virus/T7 RNA polymerase system was used for heterologous expression of the mutants and has been described in detail previously (Blakely et al., 1991). Briefly, HeLa cells were seeded in $75 \mathrm{~cm}^{2}$ cell culture flasks and allowed to grow to confluency. The confluent cells were infected with recombinant VTF7-3 virus and transfected with plasmid containing rSERT cDNA under control of the T7 promoter. At $24 \mathrm{~h}$ after transfection, the cells were rinsed with room temperature binding buffer [10 mM HEPES/150 mM NaCl adjusted to $\mathrm{pH} 8.0$ with $N$-methyl-Dglucamine (NMDG)], scraped, and collected with $10 \mathrm{ml}$ of binding buffer containing $0.5 \%$ protease inhibitor mixture (Sigma) and $100 \mu \mathrm{M}$ phenylmethylsulfonyl fluoride. Subsequently, the cells were lysed by two cycles of sonication and freeze thawing. The crude membranes were collected by centrifugation at 11,000 rpm (SS34 rotor; Sorvall) for $20 \mathrm{~min}$ at $4^{\circ} \mathrm{C}$, and the pellet was resuspended thoroughly in $1 \mathrm{ml}$ of the same buffer. Membranes were stored at $-80^{\circ} \mathrm{C}$ until used.

Binding assays. All binding assays were performed in Multiscreen-FB 96-well filtration plates (Millipore), which were preincubated with $0.1 \%$ polyethyleneimine ( $100 \mu \mathrm{l} /$ well). Before adding the membrane samples, the plates were rinsed three times with $100 \mu \mathrm{l}$ of room temperature binding buffer. Four different binding buffers were used in this study, as indicated (in mM): $10 \mathrm{HEPES} / 150 \mathrm{NaCl}, 10 \mathrm{HEPES} / 150$ sodium isethionate (2-hydroxyethanesulfonic acid, monosodium salt) $\left(\mathrm{Cl}^{-}\right.$-free), 10 HEPES/150 NMDG-Cl ( $\mathrm{Na}^{+}$-free), and 10 HEPES/150 NMDGdiatrizoate ( $\mathrm{NaCl}$-free).

Crude membranes, prepared as described above, were thawed on ice and diluted in one of the above binding buffers depending on the desired ionic content. One hundred microliter samples of the membrane suspension were added to each well and were washed three times by filtration with $100 \mu \mathrm{l}$ of the same buffer. Binding was initiated by addition of $100 \mu \mathrm{l}$ of binding buffer containing the indicated concentration range of each inhibitor and $0.15 \mathrm{~nm}\left[{ }^{125} \mathrm{I}\right] \beta$-CIT (RTI-55; PerkinElmer Life and Analytical Sciences) and was allowed to proceed for $1 \mathrm{~h}$ at room temperature with gentle shaking. The reaction was stopped by washing all wells with $100 \mu \mathrm{l}$ of ice-cold binding buffer. The filters were removed from the plate, transferred to Wallac 96-well Isoplates (part number 6005070) soaked with $150 \mu$ l of Optifluor and counted with Wallac Microbeta plate counter.

S277C accessibility assays. To determine the conformational effects of inhibitors, we measured their influence on MTSEA inactivation of $\left[{ }^{125} \mathrm{I}\right] \beta$-CIT binding to membranes of the SERT mutant S277C (Zhang and Rudnick, 2006). This mutant was generated in the $\mathrm{X} 5 \mathrm{C}$ background [SERT mutant in which the five most reactive cysteines were mutated (C15A/C21A/C109A/C357I/C622A)] (Sato et al., 2004). The cysteine at position 277 becomes accessible when the cytoplasmic permeation pathway opens (Zhang and Rudnick, 2006). The membranes were applied as above to Multiscreen-FB plates and preincubated with cocaine, ibogaine, imipramine, or fluoxetine (as indicated) for $10 \mathrm{~min}$ at room temperature with gentle shaking. Subsequently, the medium was replaced with binding buffer (10 mM HEPES, pH 8.0 with $150 \mathrm{~mm}$ salt as indicated), containing MTSEA and the inhibitor (at the same concentration as in the preincubation). After $15 \mathrm{~min}$ of incubation, the reaction with MTSEA was terminated by washing the membranes five times with binding buffer to make sure that MTSEA and the inhibitors were removed. Residual $\beta$-CIT binding was determined as described above.
Data analysis. Nonlinear regression fits of experimental data were performed with Origin (OriginLab), which uses the Marquardt-Levenberg nonlinear least-squares curve-fitting algorithm. Statistical analysis comparing multiple independent experiments was performed in Origin8 using either a two-sample $t$ test or one-way ANOVA, followed by the Tukey's multiple comparisons test as indicated. Error values were calculated as SEM using values from separate independent experiments in each case. Unless indicated otherwise, error bars in the figures are SDs for replicate measurements.

Computational analysis. Molecular models of SERT containing two $\mathrm{Na}^{+}$ions were constructed based on the extracellular-facing substratebound conformation of LeuT [Protein Data Base identification number 2a65 (Yamashita et al., 2005)], as described previously (Celik et al., 2008; Forrest et al., 2008), and were energy minimized to a maximum root mean square deviation of $0.18 \AA$. Docking was performed in the presence and absence of a $\mathrm{Cl}^{-}$ion modeled at the position of E290 from LeuT (Forrest et al., 2008).

Molecular models of imipramine, fluoxetine, paroxetine, and cocaine, with parameters generated using MacroModel 9.5 (Schrödinger), were then docked into the SERT models using the InducedFit protocol (Schrödinger) (Sherman et al., 2006). Evidence of competitive inhibition for, e.g., sertraline, citalopram, paroxetine, and imipramine (Talvenheimo et al., 1979; Thomas et al., 1987; Koe et al., 1990; Apparsundaram et al., 2008), suggests that they either share the same binding site with 5-HT or that their binding sites overlap. This so-called S1 site overlaps with the leucine binding site in LeuT. However, some evidence suggests that antidepressants bind to a second site in the extracellular permeation pathway of LeuT (Singh et al., 2007; Zhou et al., 2007, 2009). The possibility that a second molecule of substrate binds at this S2 site has also been proposed (Shi et al., 2008). Thus, compounds were docked to the region of either S1 or S2 site in the SERT model. The InducedFit protocol consisted of three stages.

First, multiple conformations and orientations of the ligand in the binding site were docked using Glide version 4.5 (Schrödinger) (Friesner et al., 2004; Halgren et al., 2004) and screened using the SP scoring function with a softened van der Waals potential (scaled down by a factor of two). In separate calculations, a Phe-341 to alanine mutation was introduced during docking of all ligands to the S1 site to maximize the search space in this step: Phe-341 is not conserved in the NSS family, and its conformation significantly impacts the shape of the binding pocket in the SERT models used.

Second, the protein structure was refined around these initial docked conformations; side-chains within $5 \AA$ of the drug (including Phe-341) were rebuilt, refined, and energy minimized with the ligand using Prime version 1.6 (Schrödinger). Up to 20 protein-ligand complexes from this step, with energies within $30 \mathrm{kcal} / \mathrm{mol}$ of the lowest-energy conformation, were retained. This refinement step was also repeated, while allowing adaptability of nearby uncoiled main-chain regions, using Prime loop refinement immediately before the side-chain refinement step. The flexible main-chain regions in S1 were from TM1 and TM6 (residues 96-99 and 337-343, respectively) and in S2 consisted of the EL4a-EL4b loop (residues 397-402) and the EL5 loop between TM9 and TM10 (residues 484-489).

Third, the ligand was docked back into the newly optimized protein. The final poses were clustered using the average-linkage clustering program NMRCLUST (Kelley et al., 1996).

Electrostatic interaction energies between the $\mathrm{Cl}^{-}$ion and the inhibitor were calculated as the product of the partial charges of the drug and the potential generated by the $\mathrm{Cl}^{-}$ion, in the presence of the dielectric environment of the protein, drug, ions, and a low-dielectric slab mimicking the membrane. Electrostatic potentials were calculated with DelPhi version 4 (Rocchia et al., 2002), as described previously (Forrest et al., 2007), and atomic charges for the drugs were generated with MacroModel.

\section{Results}

Chloride modulates antidepressant binding to SERT

Previous observations demonstrated that imipramine binding to SERT was stimulated by $\mathrm{Cl}^{-}$(Humphreys et al., 1994), whereas 
Table 1. $K_{1}$ values for displacement of $\beta$-CIT from wild-type SERT

\begin{tabular}{lcccl}
\hline & $\mathrm{NaCl}$ & Na-Ise & NMDG-Cl & NMDG-diatrizoate \\
\hline $\boldsymbol{\beta}$-CIT & $\mathbf{0 . 6} \pm \mathbf{0 . 2}$ & $\mathbf{0 . 6} \pm \mathbf{0 . 2}$ & $\mathbf{2 . 6} \pm \mathbf{0 . \mathbf { 4 } ^ { * }}$ & $\mathbf{2 . 5} \pm \mathbf{0 . \mathbf { 8 } ^ { * }}$ \\
Cocaine & $486 \pm 44$ & $405 \pm 27$ & $1657 \pm 47^{*}$ & $\mathrm{ND}$ \\
Paroxetine & $2.0 \pm 0.4$ & $2.0 \pm 0.8$ & $6.4 \pm 1.1^{*}$ & $\mathrm{ND}$ \\
Imipramine & $61 \pm 4$ & $221 \pm 13^{*}$ & $296 \pm 36^{*}$ & $518 \pm 69^{*}$ \\
Fluoxetine & $13 \pm 1$ & $38 \pm 0.8^{*}$ & $48 \pm 3^{*}$ & $66 \pm 8^{*}$ \\
Sertraline & $4.2 \pm 0.7$ & $7.0 \pm 1.3^{*}$ & $30 \pm 3^{*}$ & ND \\
Citalopram & $3.1 \pm 0.5$ & $10.5 \pm 3.2^{*}$ & $16 \pm 2^{*}$ & ND \\
lbogaine & $3402 \pm 387$ & $2592 \pm 518$ & $1112 \pm 213^{*}$ & ND
\end{tabular}

Displacement of $\beta$-CIT binding from wild-type rSERT was performed as described for Figure 1 under four ionic conditions [NaCl, Na-isethionate (Na-Ise), NMDG-Cl, and NMDG-diatrizoate]. $K_{D}$ values (in bold) (in nanomolar concentrations) were calculated for $\beta$-CIT binding and were used to determine $K_{1}$ values (in nanomolar concentrations) of the other inhibitors using the Cheng-Prusoff equation (Cheng and Prusoff, 1973). ND, Not determined. Statistical analysis from three or four independent experiments ( 2 for $\mathrm{Na}^{+}$dependences published previously; paroxetine, fluoxetine, and citalopram in NMDG-Cl) was performed in Origin8 using one-way ANOVA, followed by Tukey's multiple comparisons test. ${ }^{*} p<0.03$, statistically significant differences between affinities in $\mathrm{NaCl}$ versus the other ionic condition. The difference between NMDG-Cl and NMDG-diatrizoate was statistically significant for imipramine $(p=0.006)$ but not for fluoxetine $(p=0.06)$.

binding of other inhibitors, such as cocaine and paroxetine, was not (Cool et al., 1990; Rudnick and Wall, 1991; Wall et al., 1993). Sodium, however, stimulated binding of all tested inhibitors (Cool et al., 1990; Humphreys et al., 1994). To further our understanding of the role of ions in binding, we tested the effect of $\mathrm{Cl}^{-}$and $\mathrm{Na}^{+}$on binding of a set of SERT inhibitors, including the tricyclic antidepressant imipramine, the antidepressants fluoxetine, sertraline, citalopram, and paroxetine, and also cocaine, its analog $\beta$-CIT, and ibogaine. Several studies support the proposal that all these compounds bind in the substrate site on neurotransmitter transporters (Beuming et al., 2008; Andersen et al., 2009), although others suggest that a secondary site is involved (Zhou et al., 2007, 2009). In either case, inhibitor binding is believed to completely block transport function.

Binding affinity was determined by competition with $\left[{ }^{125} \mathrm{I}\right] \beta-$ CIT binding to membranes from HeLa cells expressing wild-type (WT) SERT. $K_{\mathrm{I}}$ values were measured in the presence and absence of $\mathrm{Na}^{+}$or $\mathrm{Cl}^{-}$, replacing $\mathrm{Na}^{+}$with the cation NMDG and $\mathrm{Cl}^{-}$with isethionate or diatrizoate. We first calculated $K_{\mathrm{D}}$ values for $\beta$-CIT in all four possible ionic conditions (Table 1 ). The $K_{\mathrm{D}}$ for $\beta$-CIT binding was unaffected by the absence of $\mathrm{Cl}^{-}$but was increased fourfold by removing $\mathrm{Na}^{+}$(Table 1). Because the $\mathrm{Na}^{+}$ dependence of $\beta$-CIT affinity could influence the ability of other ligands to displace this radioligand, we used a concentration of $\left[{ }^{125} \mathrm{I}\right] \beta$-CIT $(0.15 \mathrm{~nm})$ well below the $K_{\mathrm{D}}$ for binding $(0.6-2.6$ $\mathrm{nM})$. Moreover, we corrected for the small change in $\beta$-CIT affinity using the Cheng-Prusoff equation (Cheng and Prusoff, 1973).

The effect of $\mathrm{Na}^{+}$on binding is shown in Figure 1 and Table 1. Consistent with previous studies, the potency for most of the ligands tested was reduced in the absence of $\mathrm{Na}^{+} . K_{\mathrm{I}}$ values for cocaine, $\beta$-CIT, imipramine, fluoxetine, paroxetine, sertraline, and citalopram increased approximately threefold to sevenfold in NMDG-Cl relative to $\mathrm{NaCl}$. The sole exception was ibogaine, an inhibitor that stabilizes the cytoplasmic-facing conformation of SERT (Jacobs et al., 2007), which was more potent in the absence of $\mathrm{Na}^{+}\left(K_{\mathrm{I}}\right.$ of $3.4 \pm 0.4$ and $1.1 \pm 0.2 \mu \mathrm{M}$ in NaCl and NMDG-Cl, respectively).

We then tested the $\mathrm{Cl}^{-}$dependence of the same set of inhibitors. The data in Figure 2 and Table 1 show that fluoxetine, sertraline, and citalopram, like imipramine, bind more tightly in the presence of $\mathrm{Cl}^{-}$. The increase in affinity ranged from 1.7-fold for sertraline to 3.6-fold for imipramine. In agreement with previous studies, affinities for cocaine, $\beta$-CIT, and paroxetine were unaffected by $\mathrm{Cl}^{-}$(Cool et al., 1990; Humphreys et al., 1994). We also tested binding of $\beta$-CIT, imipramine, and fluoxetine in the absence of $\mathrm{NaCl}$ using NMDG-diatrizoate as a replacement. Although $\beta$-CIT binding was similar in NMDG-diatrizoate and NMDG-Cl, imipramine bound with reduced affinity in $\mathrm{NaCl}-$ free medium, indicating that each ion independently increased affinity for this antidepressant.

\section{Mutation of proposed $\mathrm{Cl}^{-}$binding site residues alters the $\mathrm{Cl}^{-}$ dependence of antidepressant binding}

We recently proposed a binding site that mediates the $\mathrm{Cl}^{-}$dependence of neurotransmitter transport. Mutations at these positions altered the $\mathrm{Cl}^{-}$dependence of 5-HT transport and in some cases removed the requirement for $\mathrm{Cl}^{-}$(Forrest et al., 2007). To determine whether this same $\mathrm{Cl}^{-}$site is responsible for the $\mathrm{Cl}^{-}$dependence of inhibitor binding, we characterized several mutants at positions Ser-372, Asn-368, Ser-336, and Tyr-121 that were predicted to coordinate $\mathrm{Cl}^{-}$(Forrest et al., 2007). We converted these positions to either aspartic acid, which might be expected to substitute for the negatively charged $\mathrm{Cl}^{-}$ion, or to aliphatic residues (alanine for Ser-372 and Ser-336, leucine or alanine for Asn-368, and leucine for Tyr-121 because smaller replacements at 121 were not expressed well), to prevent that position from coordinating chloride ions. Introduction of aspartate or glutamate at position Tyr-121 eliminated $\beta$-CIT binding and those mutants were not further examined.

Using the above mutants, we measured the inhibitory potencies of two antidepressants, fluoxetine and imipramine, that bound more tightly in the presence of $\mathrm{Cl}^{-}$, and cocaine, which was not affected by $\mathrm{Cl}^{-}$. The results are shown in Figure 3 and Table 2. Any mutation, to either aspartic acid or an aliphatic residue, at $121,336,368$, or 372 eliminated the ability of $\mathrm{Cl}^{-}$to increase fluoxetine or imipramine affinity. For both antidepressants, aliphatic substitutions led to marked reductions in binding. For example, fluoxetine affinities in $\mathrm{NaCl}$ were reduced to 11, 13 , and $15 \%$ of wild-type affinity, respectively, in N368L, S372A, and S336A. Significantly, when aspartic acid was introduced at positions S336 or S372, the affinity remained high (90 \pm 11 and $56 \pm 2 \%$ of WT, respectively) or became even higher than WT in the case of N368D ( $350 \pm 50 \%$ of WT). Replacement of Tyr-121 with phenylalanine or leucine or Asn-368 with alanine had an intermediate effect, decreasing fluoxetine affinity to $36 \pm 3,43 \pm$ 2 , and $40 \pm 4 \%$ of wild type, respectively. These mutations had generally the same effects on imipramine binding and its $\mathrm{Cl}^{-}$ dependence, the largest difference being that imipramine, but not fluoxetine, affinity was markedly reduced in S336D (Table 2). In contrast to mutations at $121,336,368$, or 372 , mutation of Cys369 to alanine or serine did not affect $\mathrm{Cl}^{-}$stimulation (Table 2). Cys-369 corresponds to a residue proposed to participate in a $\mathrm{Cl}^{-}$site in GAT-1 (Zomot et al., 2007).

Most mutations that had an impact on fluoxetine and imipramine affinity had only a minor influence on cocaine binding (Fig. 3, Table 2), which was not stimulated by $\mathrm{Cl}^{-}$. The only mutations that affected cocaine binding were S336D, for which affinity was reduced to $30 \pm 2 \%$ of WT (similar to imipramine), and N368D, which increased cocaine affinity 3.5-fold. These results indicate that the ability of chloride to stimulate binding requires the same binding site residues proposed to mediate the $\mathrm{Cl}^{-}$requirement for transport (Forrest et al., 2007).

\section{Flexible-fit docking suggests that the effect of $\mathrm{Cl}^{-}$ions on antidepressant binding is unlikely to be direct}

To test the possibility that $\mathrm{Cl}^{-}$stimulates antidepressant binding through a direct interaction, we used molecular modeling and 
docking approaches. Specifically, we predicted the conformations of the tested compounds bound to a homology model of SERT based on LeuT in an extracellularfacing substrate-occluded conformation (Yamashita et al., 2005). Compounds were docked to either the central S1 binding site that overlaps the leucine in LeuT or the secondary S2 binding site in the extracellular vestibule (Singh et al., 2007; Zhou et al., 2007; Shi et al., 2008). In the S1 site, the large majority of poses generated for all compounds contained a close interaction between the side-chain carboxyl of Asp-98 and the charged amine of the ligand (Fig. 4). This positioning of the amine close to Asp-98 is consistent with experimental evidence (Barker et al., 1999; Celik et al., 2008), as well as with docking results for 5-HT to SERT (Celik et al., 2008; Kaufmann et al., 2009) and for dopamine, CFT, and cocaine to DAT (Beuming et al., 2008).

In this arrangement, the amine group was the closest part of the ligand to the $\mathrm{Cl}^{-}$binding site, albeit separated by the $\mathrm{Nal}$ binding site. The mean distance of the position of the $\mathrm{Cl}^{-}$ion to the positively charged amine nitrogen atom of the ligand was $\sim 10 \AA$ for fluoxetine, imipramine, cocaine, and paroxetine (Table 3 ). These distances suggest that the only possible direct interaction between $\mathrm{Cl}^{-}$and the drug is a relatively weak, favorable electrostatic interaction. Indeed, the electrostatic interaction energy with the $\mathrm{Cl}^{-}$ion, calculated using the PoissonBoltzmann equation and averaged over all chloride-bound poses, was similarly small for each drug (Table 3). Using a protein dielectric constant of 4 , which reflects moderate screening by the protein atoms, the interaction energies are approximately $-5 \mathrm{kcal} / \mathrm{mol}$, whereas using a dielectric of 8, which might also be considered reasonable, these energies are approximately $-2.5 \mathrm{kcal} / \mathrm{mol}$. The differences in electrostatic interaction energies between the drugs were statistically significant $(p<0.001$ with a two-tailed $t$ test over all low-energy poses) only between cocaine and either fluoxetine or paroxetine.

In contrast to the results for the primary site, no clear preferences for specific orientations were observed for the ligands docked to the secondary site (data not shown). However, the mean distance of the position of the $\mathrm{Cl}^{-}$ion to the positively charged amine nitrogen atom of the ligand in S2 was consistently in the range 17-20 $\AA$ for all compounds tested (Table 3 ). This is similar to the distance of $16.4 \pm 0.1 \AA$ between the amine nitrogen and Glu-290 determined for structures of LeuT with tricyclic antidepressants bound to S2 (Singh et al., 2007; Zhou et al., 2007). Consistent with these large distances, the electrostatic energies of interaction of the ligands with
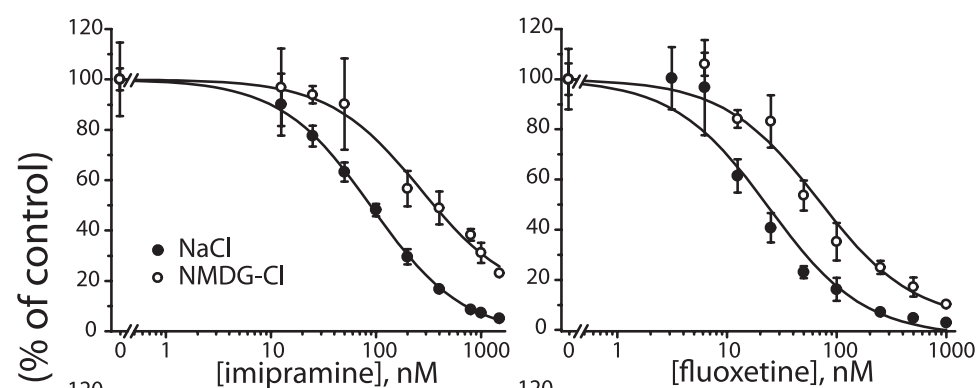

Figure 1. Displacement of $\beta$-CIT binding to wild-type rat SERT by cocaine, ibogaine, imipramine, and fluoxetine in the presence or absence of $\mathrm{Na}^{+}$. Membranes from HeLa cells expressing rSERT were incubated with $0.15 \mathrm{~nm}$ [ $\left.{ }^{125} \mathrm{I}\right] \beta$-CIT and the indicated concentrations of cocaine $(0-20 \mu \mathrm{m})$, ibogaine $(0-80 \mu \mathrm{m})$, imipramine $(0-1.5 \mu \mathrm{M})$, and fluoxetine $(0-1 \mu \mathrm{m})$ in either $150 \mathrm{~mm}$ $\mathrm{NaCl}$ (filled circles) or $150 \mathrm{~mm} N M D G-C l$ (open circles). The concentration of $\beta$-CIT used ( $0.15 \mathrm{~nm}$ ) was below the $K_{D}$ for binding in NMDG-CI (2.6 nM), so as to minimize the effect of the lower $\beta$-CIT affinity in the absence of $\mathrm{Na}^{+}$. Representative results are presented as the percentage of $\left[{ }^{125} \mathrm{I}\right] \beta$-CIT bound in the absence of the inhibitor. $K_{\mathrm{I}}$ values calculated from multiple experiments and the corresponding statistical analyses are presented in Table 1.
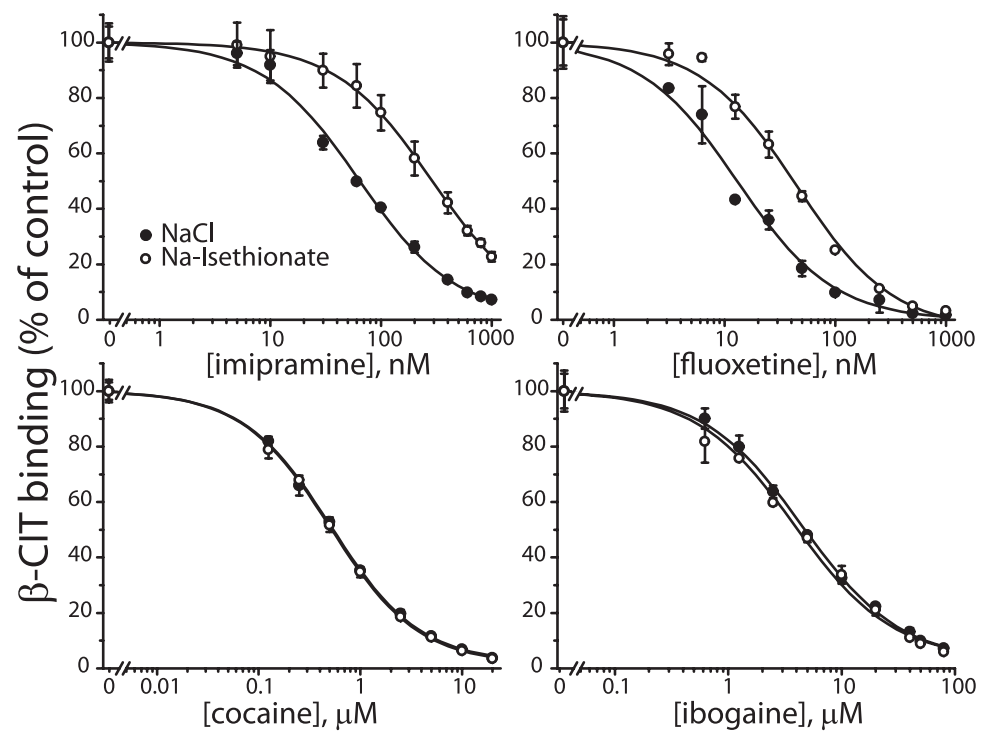

Figure 2. Displacement of $\beta$-CIT binding to SERT by cocaine, ibogaine, imipramine, and fluoxetine in the presence or absence of $\mathrm{Cl}^{-}$. The effect of $\mathrm{Cl}^{-}$on displacement of $\beta$-CIT was tested in membranes from HeLa cells expressing wild-type rSERT. The membranes were incubated with $0.15 \mathrm{~nm}\left[{ }^{125} \mathrm{I}\right] \beta$-CIT and the indicated concentrations of cocaine $(0-20 \mu \mathrm{m})$, ibogaine $(0-80$ $\mu \mathrm{M})$, imipramine $(0-1 \mu \mathrm{M})$, and fluoxetine $(0-1 \mu \mathrm{m})$ in either $150 \mathrm{~mm} \mathrm{NaCl}$ (filled circles) or $150 \mathrm{~mm} \mathrm{Na}$-isethionate (open circles). Representative results are presented as percentage of $\left[{ }^{125} \mathrm{I}\right] \beta$-CIT bound in the absence of the inhibitor. $K_{\mathrm{I}}$ values calculated from multiple experiments and the corresponding statistical analyses are presented in Table 1.

the $\mathrm{Cl}^{-}$ion were small (less than or equal to $-1 \mathrm{kcal} / \mathrm{mol}$ ), with statistically significant differences between cocaine and either fluoxetine or paroxetine, and between imipramine and fluoxetine.

Thus, no clear distinction could be made between those compounds that are affected by $\mathrm{Cl}^{-}$, such as fluoxetine and imipramine, and those that are not, such as cocaine and paroxetine, in terms of the docking results or electrostatic interaction energies. Consequently, a direct electrostatic interaction within this 


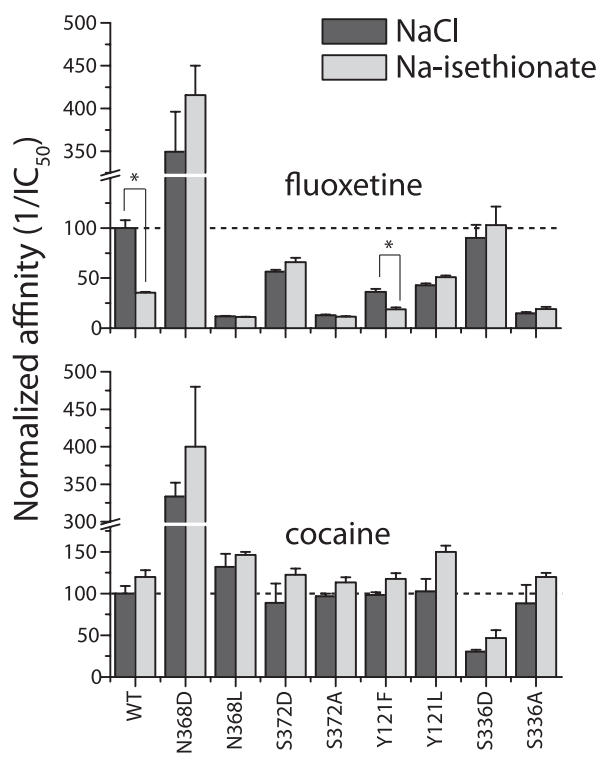

Figure 3. Effect of mutations at the chloride binding site on cocaine and fluoxetine affinity. Binding affinity for fluoxetine (top) and cocaine (bottom) for mutants of Tyr-121, Ser-336, Asn-368, and Ser-372 was determined through displacement of $\beta$-CIT binding in the presence and absence of chloride. Crude membranes from cells expressing these mutants were incubated in $150 \mathrm{~mm} \mathrm{NaCl}$ (darker bars) or $150 \mathrm{~mm} \mathrm{Na}$-isethionate (lighter bars) in the presence of $0.15 \mathrm{~nm}$ $\left[{ }^{125} \mathrm{I}\right] \beta$-CIT and increasing concentrations of cocaine $(0-20 \mu \mathrm{m})$ or fluoxetine $(0-1 \mu \mathrm{M})$. Inhibitor concentrations resulting in half-maximal inhibition of $\beta$-CIT binding were calculated and the corresponding affinities $\left(1 / \mathrm{IC}_{50}\right)$ were plotted as percentage of binding affinity for WT SERT in the presence of $150 \mathrm{~mm} \mathrm{NaCl}$. Error bars correspond to \pm SE from two to four independent experiments. Asterisks indicate a statistically significant increase in affinity in $\mathrm{NaCl}$ relative to Na-isethionate.

Table 2. Effect of $\mathrm{Cl}^{-}$on ligand binding affinity for wild-type and mutant SERT

\begin{tabular}{|c|c|c|c|c|c|c|}
\hline \multirow[b]{2}{*}{$\mathrm{Cl}^{-}$} & \multicolumn{2}{|l|}{ Cocaine (nм) } & \multicolumn{2}{|c|}{ Fluoxetine (nm) } & \multicolumn{2}{|c|}{ Imipramine (nм) } \\
\hline & + & - & + & - & + & - \\
\hline WT & $486 \pm 44$ & $405 \pm 27$ & $13 \pm 1$ & $38 \pm 1^{\dagger}$ & $61 \pm 4$ & $221 \pm 13^{\dagger}$ \\
\hline Y121F & $494 \pm 16$ & $413 \pm 24$ & $36 \pm 3^{*}$ & $71 \pm 7^{\dagger}$ & $223 \pm 9 *$ & $322 \pm 3^{\dagger}$ \\
\hline Y121L & $474 \pm 69$ & $324 \pm 16$ & $32 \pm 2^{*}$ & $26 \pm 1$ & $160 \pm 31^{*}$ & $147 \pm 23$ \\
\hline S336A & $551 \pm$ & $405 \pm 16$ & $91 \pm 9^{*}$ & $70=$ & $744 \pm 71^{*}$ & $564 \pm 9$ \\
\hline S336D & $1596 \pm 113^{*}$ & $1037 \pm 202$ & $14 \pm 2$ & $13 \pm 2$ & $699 \pm 141$ & $513 \pm 91$ \\
\hline N368A & ND & ND & $33 \pm 5^{*}$ & $29 \pm 4$ & $183 \pm 31^{*}$ & $179 \pm 47$ \\
\hline N368D & $146 \pm 8^{*}$ & & $3.8 \pm$ & $3.2 \pm 0.2$ & $15 \pm 3^{*}$ & \pm 2 \\
\hline N368L & $369 \pm 44$ & $332 \pm 8$ & $113 \pm 5^{*}$ & $120 \pm 3$ & $1375 \pm 84^{*}$ & $1158 \pm 157$ \\
\hline C369A & ND & ND & $20 \pm 1^{*}$ & $41 \pm 4^{\dagger}$ & $144 \pm 41^{*}$ & $329 \pm 65^{\dagger}$ \\
\hline C369S & ND & ND & $25 \pm 1$ & $75 \pm 7^{\dagger}$ & $40 \pm 2$ & $213 \pm 3^{\dagger}$ \\
\hline S372A & $502 \pm 17$ & $429 \pm 24$ & $105 \pm 8^{*}$ & $117 \pm 5$ & $522 \pm 138$ & $480 \pm 55$ \\
\hline S372D & $547 \pm 142$ & $397 \pm 24$ & $23 \pm 1^{*}$ & $20 \pm 2$ & $245 \pm 32^{*}$ & $215 \pm 21$ \\
\hline
\end{tabular}

$K_{1}$ values for displacement of $\beta$-CIT binding by cocaine, imipramine, and fluoxetine were determined in the presence and absence of $\mathrm{Cl}^{-}$, as in Table 1, for mutants at proposed chloride binding site residues and Cys-369 as a control (in bold). *Significant differences $(p<0.05)$ between wild-type and mutants in NaCl medium. 'Statistically significant differences $(p<0.05)$ between $\mathrm{NaCl}$ and Na-isethionate for a given mutant. Statistical analysis is from three to five independent experiments ( 2 when the difference between wild-type and mutant was $>8$-fold: imipramine binding to S336A, D, N368L, and S372A). ND, Not determined. Analysis was performed in Origin8 using the two-sample $t$ test.

extracellular-facing conformation of SERT appears not to be sufficient to explain the markedly different $\mathrm{Cl}^{-}$effects on binding.

\section{Fluoxetine binding stabilizes SERT in a novel conformational state}

$\mathrm{Cl}^{-}$was shown previously to allow 5-HT-induced conformational changes in SERT (Zhang and Rudnick, 2006). We considered the possibility that its role in antidepressant binding is to facilitate conformational transitions that allow these compounds to bind with maximal affinity. Previously, we showed that accessibility of residues in the cytoplasmic permeation pathway was altered by ligand binding. To examine whether antidepressant binding alters the conformational state of SERT, we tested the effect of imipramine and fluoxetine on the reactivity of a cysteine at position 277 in the cytoplasmic permeation pathway. This residue is on the face of TM5 that contributes to the pathway, and its reactivity was shown to increase and decrease in response to ligands (Zhang and Rudnick, 2006; Jacobs et al., 2007). Furthermore, it is close to the cytoplasmic end of TM5, a position where it is unlikely to be directly affected by ligand binding at the substrate site.

We measured the ability of MTSEA to inactivate $\beta$-CIT binding to membranes prepared from cells expressing SERT S277C (Fig. 5). Under the conditions used, $0.1 \mathrm{~mm}$ MTSEA inactivated approximately half of the $\beta$-CIT binding in the absence of added ligands. Cocaine decreased this inhibition, resulting in more residual $\beta$-CIT binding activity, consistent with the ability of cocaine to stabilize the extracellular-facing conformation of SERT, in which the cytoplasmic pathway is closed. Ibogaine, in contrast, increased the extent of inactivation, leading to less residual activity. Ibogaine has been proposed to stabilize the cytoplasm-facing conformation of SERT, in which the cytoplasmic pathway is open (Jacobs et al., 2007). Fluoxetine and imipramine led to levels of inactivation intermediate between those measured with cocaine or ibogaine. Figure 5 shows that, even at saturating concentrations, the antidepressants affected the reactivity of S277C in a quantitatively different manner than either cocaine or ibogaine. In separate experiments, we found that $\mathrm{Cl}^{-}$affected the concentrations of imipramine and fluoxetine required to influence MTSEA sensitivity, although at saturating concentrations, they had the same effect in the presence or absence of $\mathrm{Cl}^{-}$. The imipramine effect on MTSEA inactivation required approximately eightfold higher concentrations of imipramine in the absence of $\mathrm{Cl}^{-}$(Fig. 5, inset). Because the change in MTSEA sensitivity with fluoxetine was small, it was difficult to accurately measure the effect of $\mathrm{Cl}^{-}$on fluoxetine potency.

The same ligands were then tested at saturating concentrations over a range of MTSEA concentrations so as to more precisely determine their effect on S277C reactivity (Fig. 6). The MTSEA concentration leading to half-maximal inactivation provides a measure of reactivity under each condition (Rudnick, 2002). The results show that fluoxetine (300 nM), imipramine (1 $\mu \mathrm{M})$, and cocaine $(10 \mu \mathrm{M})$ all decreased the reactivity of S277C relative to MTSEA alone. Each of these inhibitors increased the MTSEA concentration required for half-maximal inactivation, whereas ibogaine $(20 \mu \mathrm{M})$ decreased that concentration, indicating higher reactivity of S277C. Significantly, the effect of fluoxetine was markedly different from that of cocaine, decreasing the rate constant for S277C modification fourfold, whereas cocaine and imipramine decreased it by 12 -fold and 6-fold, respectively (see legend of Fig. 6). We interpret the changes in inactivation rate to indicate that saturating concentrations of fluoxetine and possibly imipramine result in a different conformational equilibrium than cocaine and that those conformational preferences led to different reactivities of $\mathrm{S} 277 \mathrm{C}$ with each of the three ligands bound.

\section{Discussion}

As inhibitors of 5-HT reuptake, antidepressants acting on SERT represent an important therapeutic tool. To understand how these compounds act and to guide the development of new antidepressants, it is essential to understand the structural and mechanistic details underlying the binding process. The work presented here was undertaken to characterize the structural and 
mechanistic aspects of antidepressant binding to SERT in the light of advances in our understanding of SERT structure and function.

We show here that, in addition to imipramine, fluoxetine, sertraline, and citalopram, which are among the most widely used antidepressants, all bind more avidly to SERT in the presence of $\mathrm{Cl}^{-}$. Although the affinities of cocaine and the cocaine analog $\beta$-CIT were not stimulated by $\mathrm{Cl}^{-}$, all but one of the drugs tested bound with higher affinity in the presence of $\mathrm{Na}^{+}$(Table 1). An interesting exception was ibogaine, which is proposed to stabilize the cytoplasm-facing conformation of SERT (Jacobs et al., 2007) and which bound with increased affinity when $\mathrm{Na}^{+}$was removed (Table 1 ). We speculate that this inverse effect may be related to the uniquely different conformation to which ibogaine binds. If so, it would be consistent with evidence that $\mathrm{Na}^{+}$binding favors the extracellular-facing conformation of transporters in this family (Chen and Reith, 2003; Quick et al., 2006).

We recently identified a $\mathrm{Cl}^{-}$ion binding site in SERT (Forrest et al., 2007) common to $\mathrm{Cl}^{-}$-dependent neurotransmitter transporters, including GAT-1 (Zomot et al., 2007). The work presented here supports the participation of the same site in antidepressant binding. The ability of the proposed $\mathrm{Cl}^{-}$ site residues to coordinate $\mathrm{C} \mathrm{Cl}^{-}$ion depends on their polar character. Consequently, replacing any of these residues with aliphatic amino acids eliminated the ability of $\mathrm{Cl}^{-}$to stimulate imipramine or fluoxetine binding (Fig. 3, Table 2).

We and others originally proposed that $\mathrm{Cl}^{-}$was needed to stabilize one of the $\mathrm{Na}^{+}$ions required for transport (Forrest et al., 2007; Zomot et al., 2007). In many NSS transporters that do not require $\mathrm{Cl}^{-}$, such as LeuT and TnaT (a prokaryotic tryptophan transporter), a carboxylic amino acid is found at the position corresponding to Ser-372 in SERT. In S372D and N368D, 5-HT transport became $\mathrm{Cl}^{-}$independent, although these mutants had low transport activity (Forrest et al., 2007). Mutants at these positions retained high $\beta$-CIT binding activity, however, allowing more thorough analysis of the role of this $\mathrm{Cl}^{-}$site on antidepressant affinity. For three of the four proposed binding site residues (Y121D and Y121E were inactive), replacement with aspartate led to $\mathrm{Cl}^{-}$independent fluoxetine binding with affinity close to or better than wild type (Fig. 3, Table 2), consistent with our proposal that the negatively charged carboxylate side chain could replace a bound $\mathrm{Cl}^{-}$ion (Forrest et al., 2007). We considered two ways that $\mathrm{Cl}^{-}$ could enhance binding: by direct physical interaction (either steric or electrostatic) or through conformational change.

Analysis of homology models of the outward-facing substratebound conformation of SERT indicates that any direct interaction of the $\mathrm{Cl}^{-}$ion would be limited to long-range $(>8 \AA)$ electrostatics. We performed flexible-fit docking of the drugs to these SERT homology models to determine whether, in a given state of the protein, such direct electrostatic interactions with $\mathrm{Cl}^{-}$would differ for the various drugs. The results show that any electrostatic energy gained during $\mathrm{Cl}^{-}$binding would be similar for all compounds, which is not consistent with the different effects of $\mathrm{Cl}^{-}$on binding affinity for, e.g., cocaine and fluoxetine.

Conversely, $\mathrm{Cl}^{-}$is known to be required for conformational changes induced by 5-HT, which increased (Sato et al., 2004; Zhang and Rudnick, 2006) or decreased (Mitchell et al., 2004) reactivity of cysteine residues positioned in various parts of the SERT structure. Moreover, $\mathrm{Cl}^{-}$itself increased accessibility of a cysteine at position 277 in the cytoplasmic pathway to a small but significant extent (legend of Fig. 5). Thus, to test whether a conformational change is associated with binding, we measured the effect of ligand binding on the reactivity of a cysteine residue placed in the cytoplasmic permeation pathway. The SERT mutant S277C, like other mutants containing a cysteine in this pathway, reacts with MTSEA at rates that depend on the nature of bound ligands (Zhang and Rudnick, 2006; Forrest et al., 2008). Cocaine is believed to bind to a conformation of SERT, NET, and DAT in which the substrate binding site faces the extracellular medium and the cytoplasmic pathway is closed, leading to low reactivity of S277C (Zhang and Rudnick, 2006; Beuming et al., 2008; Forrest et al., 2008). Ibogaine, in contrast, is thought to stabilize SERT in a conformation with the binding site facing the cytoplasm, leading to an open cytoplasmic pathway and high reactivity of S277C (Jacobs et al., 2007; Forrest et al., 2008). In the 


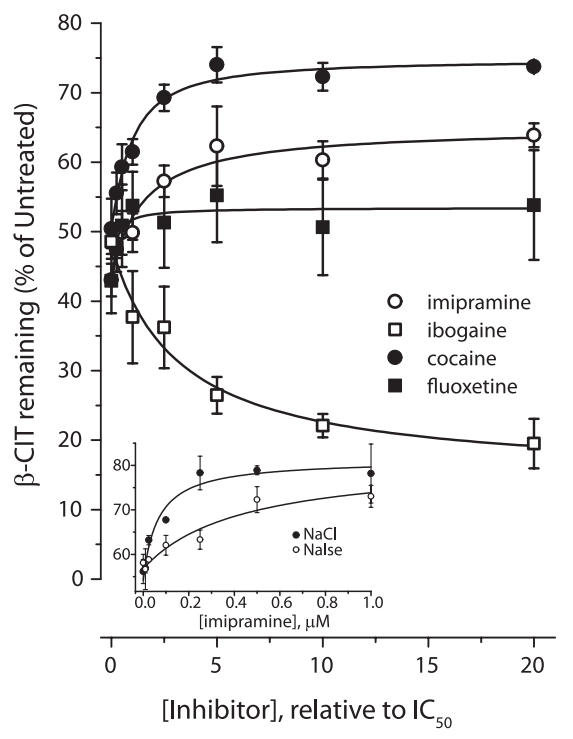

Figure 5. Modulation of MTSEA-induced inactivation of $\$ 277 \mathrm{C}$ by fluoxetine, imipramine, cocaine, and ibogaine. Membranes from cells expressing S277C were treated for 15 min with 0.1 mM MTSEA in the presence of varying concentrations of cocaine, ibogaine, imipramine, or fluoxetine. After washing membranes free of MTSEA and inhibitors, residual binding activity was determined by incubation with [ $\left.{ }^{125} \mathrm{I}\right] \beta$-CIT as described in Materials and Methods. The concentration range of each inhibitor is presented relative to its $I_{50}(x$-axis). The typical experiment illustrated was repeated four times with similar results. Inset, Imipramine effect in the presence (filled circles) or absence (open circles) of $\mathrm{Cl}^{-}$. The $\mathrm{IC}_{50}$ for imipramine was $0.4 \pm 0.1 \mu \mathrm{m}$ in the absence of $\mathrm{Cl}^{-}$and $0.05 \pm 0.02 \mu \mathrm{m}$ in its presence. In the absence of imipramine, $\mathrm{Cl}^{-}$had a minor effect, decreasing the $\mathrm{IC}_{50}$ for MTSEA by only $\sim 30 \%$ (comparing $\mathrm{NaCl}$ with Na-isethionate)

membrane preparations used here and in the absence of ligands, S277C reactivity is intermediate, probably because ligand-free SERT is in equilibrium between open and closed conformational states (Figs. 5, 6).

By saturating SERT with a ligand, we shift the equilibrium toward protein conformations optimal for binding that ligand: cytoplasm-facing for ibogaine and extracellular-facing for cocaine. Significantly, fluoxetine (and to a much lesser extent, imipramine) decreased reactivity of S277C to a level distinctly different from that of cocaine, suggesting that fluoxetine binding favors a conformation more cytoplasm facing than cocaine but not as much as with ibogaine (Figs. 5, 6). Because position 277 is near the cytoplasmic end of TM5, over $14 \AA$ from the substrate binding site, it is likely that the effect of antidepressant binding on S277 accessibility is an indirect one mediated by conformational change rather than a direct interaction. Although higher concentrations of both imipramine and fluoxetine were required to decrease S277C reactivity in the absence of $\mathrm{Cl}^{-}$, the extent of the decrease was similar with and without $\mathrm{Cl}^{-}$(Fig. 5, inset).

We recently described a mechanism for neurotransmitter transport based on conformational changes required to expose residues in the cytoplasmic permeation pathway. In this mechanism, the protein alternates between two conformations that differ in the tilt angle of a four-helix bundle composed of TM1, TM2, TM6, and TM7 (Forrest et al., 2008). The $\mathrm{Cl}^{-}$binding site residues are all contained within the bundle, suggesting that $\mathrm{Cl}^{-}$ bundle interactions are important for conformational changes induced by 5 -HT. We propose that conformational changes that alter the relationship between the bundle and the rest of the protein are influenced by $\mathrm{Cl}^{-}$binding and are required not only for alternating access in transport but also for forming the opti-

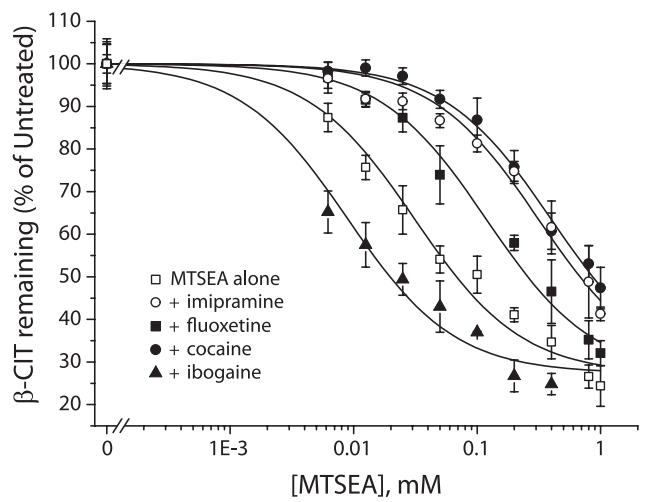

Figure 6. MTSEA dependence of SERT S277C inactivation and effects of fluoxetine, imipramine, cocaine, and ibogaine. Membranes from cells expressing S277C/X5C were incubated for 15 min with the indicated concentrations of MTSEA in the presence of saturating concentrations of cocaine (10 $\mu \mathrm{m})$, ibogaine $(20 \mu \mathrm{M})$, fluoxetine (300 nM), or imipramine $(1 \mu \mathrm{M})$. The MTSEA concentration giving half-maximal inactivation of $\left[{ }^{125} \mathrm{I}\right] \beta$-CIT binding was used to calculate inactivation rate constants. MTSEA concentrations leading to half-maximal inactivation and the corresponding rate constants were as follows: MTSEA alone, $33 \pm 4 \mu \mathrm{M}, 25 \pm 3 \mathrm{M}^{-1} \mathrm{~s}^{-1}$; cocaine, $390 \pm 43 \mu \mathrm{m}, 2 \pm 0.2 \mathrm{M}^{-1} \mathrm{~s}^{-1}$; ibogaine, $8 \pm 2 \mu \mathrm{M}, 100 \pm 15 \mathrm{M}^{-1} \mathrm{~s}^{-1}$; imipramine, $267 \pm 70 \mu \mathrm{M}, 3.9 \pm 1 \mathrm{M}^{-1} \mathrm{~s}^{-1}$; and fluoxetine, $131 \pm 24 \mu \mathrm{M}, 7 \pm 2 \mathrm{M}^{-1} \mathrm{~s}^{-1}$. Inactivation rates corresponding to four to six independent experiments were statistically analyzed in Origin8 using a two-sample $t$ test. $I C_{50}$ values for inactivation in the presence of each inhibitor were significantly different from the values with MTSEA alone. The effect of fluoxetine but not imipramine was significantly different ( $p<0.002$ for half-maximal inactivation) from that of cocaine. Nevertheless, in four of five experiments, the effect of imipramine was clearly different from that of cocaine, and in only one experiment were the effects of the two drugs indistinguishable.

mal binding conformation for fluoxetine and possibly other antidepressants.

These conclusions are consistent with the recently-reported "open-out" structure of LeuT solved in the presence of a competitive inhibitor, tryptophan (Singh et al., 2008), in which the orientation of a large portion of the bundle is altered to adopt a protein conformation more open to the extracellular side than the substrate-bound state. It also may be noteworthy that the arrangement of the residues corresponding to the proposed SERT $\mathrm{Cl}^{-}$binding site differs slightly between the substrate- and inhibitor-bound structures of LeuT. Specifically, the ability to coordinate $\mathrm{a} \mathrm{Cl}^{-}$ion may be compromised in the equivalent open-out conformation of SERT, whereas $\mathrm{Cl}^{-}$binding may act to stabilize SERT conformations that are similar to the occluded state of LeuT.

We further surmise from the different level of S277C reactivity with 5-HT, imipramine, and fluoxetine that the $\mathrm{Cl}^{-}$-dependent conformational preferences of the bundle may differ not only in extent but also direction of tilt with different ligands. For example, S277C reactivity was much greater in the presence of 5-HT compared with fluoxetine (Zhang and Rudnick, 2006 vs Fig. 6), consistent with a greater extent of tilt during transport. Additionally, although imipramine and fluoxetine binding are stimulated by $\mathrm{Cl}^{-}$to a similar extent, fluoxetine binding causes a larger increase in S277C reactivity, which may reflect a conformation with a different tilt direction.

Our results provide additional support to the notion that binding of different inhibitors influences the equilibrium between protein conformations (Zhang and Rudnick, 2006; Jacobs et al., 2007; Forrest et al., 2008). The computational results are also consistent with this idea, because by using a single protein structure it is not possible to rationalize the effect of chloride. Interestingly, several lines of evidence suggest that substrates, 
antidepressants, and psychostimulants have profound and differential effects on monoamine transporter regulation (Ramamoorthy and Blakely, 1999; Saunders et al., 2000; Daws et al., 2002; Little et al., 2002; Cervinski et al., 2005; Chanrion et al., 2007; Lau et al., 2008). It is possible that changes in the conformational equilibrium attributable to antidepressant binding might influence transporter regulation, including posttranslational modification, trafficking, and interaction of the transporter with regulatory proteins.

\section{References}

Andersen J, Taboureau O, Hansen KB, Olsen L, Egebjerg J, Strømgaard K, Kristensen AS (2009) Location of the antidepressant binding site in the serotonin transporter: importance of Ser-438 in recognition of citalopram and tricyclic antidepressants. J Biol Chem 284:10276-10284.

Apparsundaram S, Stockdale DJ, Henningsen RA, Milla ME, Martin RS (2008) Antidepressants targeting the serotonin reuptake transporter act via a competitive mechanism. J Pharmacol Exp Ther 327:982-990.

Barker EL, Moore KR, Rakhshan F, Blakely RD (1999) Transmembrane domain I contributes to the permeation pathway for serotonin and ions in the serotonin transporter. J Neurosci 19:4705-4717.

Beuming T, Kniazeff J, Bergmann ML, Shi L, Gracia L, Raniszewska K, Newman AH, Javitch JA, Weinstein H, Gether U, Loland CJ (2008) The binding sites for cocaine and dopamine in the dopamine transporter overlap. Nat Neurosci 11:780-789.

Blakely RD, Clark JA, Rudnick G, Amara SG (1991) Vaccinia-T7 RNA polymerase expression system: evaluation for the expression cloning of plasma membrane transporters. Anal Biochem 194:302-308.

Celik L, Sinning S, Severinsen K, Hansen CG, Møller MS, Bols M, Wiborg O, Schiøtt B (2008) Binding of serotonin to the human serotonin transporter. Molecular modeling and experimental validation. J Am Chem Soc 130:3853-3865.

Cervinski MA, Foster JD, Vaughan RA (2005) Psychoactive substrates stimulate dopamine transporter phosphorylation and down-regulation by cocaine-sensitive and protein kinase C-dependent mechanisms. J Biol Chem 280:40442-40449.

Chanrion B, Mannoury la Cour C, Bertaso F, Lerner-Natoli M, Freissmuth M, Millan MJ, Bockaert J, Marin P (2007) Physical interaction between the serotonin transporter and neuronal nitric oxide synthase underlies reciprocal modulation of their activity. Proc Natl Acad Sci USA 104:8119-8124.

Chen N, Reith ME (2003) $\mathrm{Na}^{+}$and the substrate permeation pathway in dopamine transporters. Eur J Pharmacol 479:213-221.

Cheng Y, Prusoff WH (1973) Relationship between the inhibition constant $\left(\mathrm{K}_{\mathrm{I}}\right)$ and the concentration of inhibitor which causes 50 per cent inhibition $\left(I_{50}\right)$ of an enzymatic reaction. Biochem Pharmacol 22:3099-3108.

Cool DR, Leibach FH, Ganapathy V (1990) High-affinity paroxetine binding to the human placental serotonin transporter. Am J Physiol 259:C196-C204.

Daws LC, Callaghan PD, Morón JA, Kahlig KM, Shippenberg TS, Javitch JA, Galli A (2002) Cocaine increases dopamine uptake and cell surface expression of dopamine transporters. Biochem Biophys Res Commun 290:1545-1550.

Forrest LR, Tavoulari S, Zhang YW, Rudnick G, Honig B (2007) Identification of a chloride ion binding site in $\mathrm{Na}^{+} / \mathrm{Cl}^{-}$-dependent transporters. Proc Natl Acad Sci U S A 104:12761-12766.

Forrest LR, Zhang YW, Jacobs MT, Gesmonde J, Xie L, Honig BH, Rudnick G (2008) Mechanism for alternating access in neurotransmitter transporters. Proc Natl Acad Sci U S A 105:10338-10343.

Friesner RA, Banks JL, Murphy RB, Halgren TA, Klicic JJ, Mainz DT, Repasky MP, Knoll EH, Shelley M, Perry JK, Shaw DE, Francis P, Shenkin PS (2004) Glide: a new approach for rapid, accurate docking and scoring. 1. Method and assessment of docking accuracy. J Med Chem 47:1739-1749.

Halgren TA, Murphy RB, Friesner RA, Beard HS, Frye LL, Pollard WT, Banks JL (2004) Glide: a new approach for rapid, accurate docking and scoring. 2. Enrichment factors in database screening. J Med Chem 47:1750-1759.

Humphreys CJ, Wall SC, Rudnick G (1994) Ligand binding to the serotonin transporter: equilibria, kinetics and ion dependence. Biochemistry 33:9118-9125.

Jacobs MT, Zhang YW, Campbell SD, Rudnick G (2007) Ibogaine, a non- competitive inhibitor of serotonin transport, acts by stabilizing the cytoplasm-facing state of the transporter. J Biol Chem 282:29441-29447.

Kaufmann KW, Dawson ES, Henry LK, Field JR, Blakely RD, Meiler J (2009) Structural determinants of species-selective substrate recognition in human and Drosophila serotonin transporters revealed through computational docking studies. Proteins 74:630-642.

Kelley LA, Gardner SP, Sutcliffe MJ (1996) An automated approach for clustering an ensemble of NMR-derived protein structures into conformationally related subfamilies. Protein Eng 9:1063-1065.

Koe BK, Lebel LA, Welch WM (1990) $\left[{ }^{3} \mathrm{H}\right]$ sertraline binding to rat brain membranes. Psychopharmacology (Berl) 100:470-476.

Lau T, Horschitz S, Berger S, Bartsch D, Schloss P (2008) Antidepressantinduced internalization of the serotonin transporter in serotonergic neurons. FASEB J 22:1702-1714.

Lingjaerde O (1969) Uptake of serotonin in blood platelets: dependence on sodium and chloride, and inhibition by choline. FEBS Lett 3:103-106.

Little KY, Elmer LW, Zhong H, Scheys JO, Zhang L (2002) Cocaine induction of dopamine transporter trafficking to the plasma membrane. Mol Pharmacol 61:436-445.

Mitchell SM, Lee E, Garcia ML, Stephan MM (2004) Structure and function of extracellular loop 4 of the serotonin transporter as revealed by cysteinescanning mutagenesis. J Biol Chem 279:24089-24099.

Murphy DL, Lerner A, Rudnick G, Lesch KP (2004) Serotonin transporter: gene, genetic disorders, and pharmacogenetics. Mol Interv 4:109-123.

Nelson PJ, Rudnick G (1979) Coupling between platelet 5-hydroxytryptamine and potassium transport. J Biol Chem 254:10084-10089.

Quick M, Yano H, Goldberg NR, Duan L, Beuming T, Shi L, Weinstein H, Javitch JA (2006) State-dependent conformations of the translocation pathway in the tyrosine transporter Tyt1, a novel neurotransmitter: sodium symporter from Fusobacterium nucleatum. J Biol Chem 281: 26444-26454.

Ramamoorthy S, Blakely RD (1999) Phosphorylation and sequestration of serotonin transporters differentially modulated by psychostimulants. Science 285:763-766.

Rocchia W, Sridharan S, Nicholls A, Alexov E, Chiabrera A, Honig B (2002) Rapid grid-based construction of the molecular surface and the use of induced surface charge to calculate reaction field energies: applications to the molecular systems and geometric objects. J Comput Chem 23:128-137.

Rudnick G (1977) Active transport of 5-hydroxytryptamine by plasma membrane vesicles isolated from human blood platelets. J Biol Chem 252:2170-2174.

Rudnick G (2002) Chemical modification strategies for structure-function studies. In: Transmembrane transporters (Quick MW, ed), pp 125-141. Hoboken, NJ: Wiley.

Rudnick G (2006) Serotonin transporters: structure and function. J Membr Biol 213:101-110.

Rudnick G, Wall SC (1991) Binding of the cocaine analog 2 beta- $\left[{ }^{3} \mathrm{H}\right]$ carboxymethoxy-3 beta-(4-fluorophenyl)tropane to the serotonin transporter. Mol Pharmacol 40:421-426.

Rudnick G, Wall SC (1992) The molecular mechanism of ecstasy [3,4methylenedioxymethamphetamine (MDMA)]: serotonin transporters are targets for MDMA-induced serotonin release. Proc Natl Acad Sci U S A 89:1817-1821.

Sato Y, Zhang YW, Androutsellis-Theotokis A, Rudnick G (2004) Analysis of transmembrane domain 2 of rat serotonin transporter by cysteine scanning mutagenesis. J Biol Chem 279:22926-22933.

Saunders C, Ferrer JV, Shi L, Chen J, Merrill G, Lamb ME, Leeb-Lundberg LM, Carvelli L, Javitch JA, Galli A (2000) Amphetamine-induced loss of human dopamine transporter activity: an internalization-dependent and cocaine-sensitive mechanism. Proc Natl Acad Sci U S A 97:6850-6855.

Sherman W, Day T, Jacobson MP, Friesner RA, Farid R (2006) Novel procedure for modeling ligand/receptor induced fit effects. J Med Chem 49:534-553.

Shi L, Quick M, Zhao Y, Weinstein H, Javitch JA (2008) The mechanism of a neurotransmitter:sodium symporter-inward release of $\mathrm{Na}^{+}$and substrate is triggered by substrate in a second binding site. Mol Cell 30:667-677.

Singh SK, Yamashita A, Gouaux E (2007) Antidepressant binding site in a bacterial homologue of neurotransmitter transporters. Nature 448:952-956. 
Singh SK, Piscitelli CL, Yamashita A, Gouaux E (2008) A competitive inhibitor traps LeuT in an open-to-out conformation. Science 322:1655-1661.

Sneddon JM (1969) Sodium-dependent accumulation of 5-hydroxytryptamine by rat blood platelets. Br J Pharmacol 37:680-688.

Talvenheimo J, Nelson PJ, Rudnick G (1979) Mechanism of imipramine inhibition of platelet 5-hydroxytryptamine transport. J Biol Chem 254:4631-4635

Thomas DR, Nelson DR, Johnson AM (1987) Biochemical effects of the antidepressant paroxetine, a specific 5-hydroxytryptamine uptake inhibitor. Psychopharmacology (Berl) 93:193-200.

Wall SC, Innis RB, Rudnick G (1993) Binding of the cocaine analog 2 $\beta$-carbomethoxy-3 $\beta$-(4-[ $\left.{ }^{125} \mathrm{I}\right]$ iodophenyl)tropane to serotonin and dopamine transporters: different ionic requirements for substrate and $2 \beta$-carbomethoxy-3 $\beta$ - $\left(4-\left[{ }^{125} \mathrm{I}\right]\right.$ iodophenyl $)$ tropane binding. Mol Pharmacol 43:264-270.

Wong DT, Bymaster FP, Engleman EA (1995) Prozac (fluoxetine, Lilly
110140), the first selective serotonin uptake inhibitor and an antidepressant drug: twenty years since its first publication. Life Sci 57:411-441.

Yamashita A, Singh SK, Kawate T, Jin Y, Gouaux E (2005) Crystal structure of a bacterial homologue of $\mathrm{Na}^{+} / \mathrm{Cl}^{-}$-dependent neurotransmitter transporters. Nature 437:215-223.

Zhang YW, Rudnick G (2006) The cytoplasmic substrate permeation pathway of serotonin transporter. J Biol Chem 281:36213-36220.

Zhou Z, Zhen J, Karpowich NK, Goetz RM, Law CJ, Reith ME, Wang DN (2007) LeuT-desipramine structure reveals how antidepressants block neurotransmitter reuptake. Science 317:1390-1393.

Zhou Z, Zhen J, Karpowich NK, Law CJ, Reith ME, Wang DN (2009) Antidepressant specificity of serotonin transporter suggested by three LeuT-SSRI structures. Nat Struct Mol Biol 16:652-658.

Zomot E, Bendahan A, Quick M, Zhao Y, Javitch JA, Kanner BI (2007) Mechanism of chloride interaction with neurotransmitter:sodium symporters. Nature 449:726-730. 